\title{
Study on measurement of sound attenuation coefficient in bubble wake by pool
}

\author{
Jing Han ${ }^{1 *}$, Shuai $\mathrm{Lv}^{1}$, Zhongpeng Wu ${ }^{1}$, Mingwei Zhang ${ }^{1}$, Jin Bai ${ }^{1}$ \\ ${ }^{1}$ Science and Technology on Underwater Test and Control Laboratory, Dalian, Liaoning, 116013, China
}

\begin{abstract}
In this paper, bubbles are generated by controlling the air inlet volume of the ceramic tube array with a gas divider valve. Stimulation tests of sound attenuation characteristics of the wake of bubbles in a laboratory pool are performed. A measurement experiment of sound attenuation coefficient was carried out in the case of still water and bubbles with different particle sizes. The signal frequency is $20-200 \mathrm{kHz}$. Through experimental research, it is found that the existence of bubbles makes the sound attenuation coefficient significantly larger. And the attenuation coefficient is related to the frequency of the sound waves and the size of the bubbles. At the same frequency, the larger the bubble size, the larger the attenuation coefficient will be. When the bubble size is constant, the attenuation of the acoustic signal in small bubbles will change greatly below $50 \mathrm{kHz}$. Above $50 \mathrm{kHz}$, the attenuation coefficient changes relatively smoothly and the fluctuation is small. In the case of medium and large bubbles, the fluctuation of the attenuation coefficient becomes larger than that in the small bubbles. Finally, the theoretically calculated sound attenuation coefficient is compared with the experimentally measured results. And the change trends of the two results are basically the same.
\end{abstract}

\section{Introduction}

Due to the disturbance of the ship's hull and propeller, a certain wake zone with bubbles and seawater co-existing will be formed on both sides and behind the ship when the ship is sailing. The bubble particle size and number density in the wake zone are related to the sailing parameters of the ship and its own scale. Air is contained in the bubble. The attenuation characteristics of air medium and seawater medium are different to sound waves. The propagation characteristics of sound waves under the mixing of seawater and bubbles are changed ${ }^{[1]}$. As a physical field that can travel long distance underwater, the propagation attenuation characteristics of sound wave are of great significance for the detection and recognition of underwater objects ${ }^{[2,3]}$. Many scholars at home and abroad have studied the bubble wake distribution and its acoustic characteristics. ${ }^{[4,5,6]}$ However, the actual measurement of sound attenuation in bubble wake at sea is not only expensive, but also difficult. For this reason, this paper adopts the simulation method to simulate the bubble wake in the laboratory pool, and then the sound attenuation coefficient in the bubble wake is measured and analysed.

\section{Introduction to the Experiment}

In the laboratory, ceramic tube array was used to generate bubbles. And nine ceramic tubes were connected in parallel to form a ceramic tube array. The aperture of the ceramic tubes was $0.1 \mathrm{um}$ and the length was $40 \mathrm{~cm}$. The test arrangement of the pool is shown in figure.1. The receiver hydrophone and the high frequency transducer were fixed on the shelves of two wagons respectively. Ceramic tube arrays were placed in the middle of the bottom of the pool. It was connected to the air compressor outside the pool by a sub-pipe. The distance between the receiving hydrophone and the highfrequency transducer was $3.7 \mathrm{~m}$. The hydrophone and the high-frequency transducer were arranged in a water depth of $1.3 \mathrm{~m}$. The size of the pool is $6 \mathrm{~m} * 3 \mathrm{~m} * 4 \mathrm{~m}$ for length, width and depth. During the experiment, the size of bubbles generated by the ceramic tube array was controlled by the air pressure valve on the sub-pipe. The high-frequency acoustic signals of static water, small bubbles, medium bubbles and large bubbles were measured in the pool respectively. The frequency of sound signal is $20-200 \mathrm{kHz}$ in this test. The sound signal form is continuous wave $(\mathrm{CW})$ and linear frequency modulation(LFM). The pulse width of the sound signal transmitted by the high frequency transducer was $1 \mathrm{~ms}$. The sound signal transmission cycle was $100 \mathrm{~ms}$. The number of processing pulses in each group would be no less than 100 . The sound signal sampling rate was $5 \mathrm{MHz}$. 


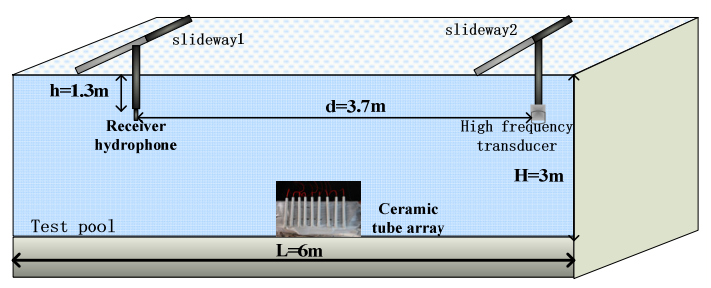

Fig.1. Experimental layout

\section{Experimental results and analysis}

During the experiment, the transducer transmits different frequency signals and uses the hydrophone to receive the acoustic signals. The frequency of acoustic signal is 20$200 \mathrm{kHz}$. The transmitted signals take the form of singlefrequency and wideband acoustic signals. The acoustic signal under the conditions of still wager, small bubbles, medium bubbles and large bubbles received by hydrophones was shown in Figure 2-5. Compared with the signals received in still water, as the bubbles grow larger, the signal amplitude gradually decreases.

The acoustic signal intensity under the conditions of still wager, small bubbles, medium bubbles and large bubbles received by hydrophones was shown in Figure 6-9. Compared with the signals received in still water, as the bubbles grow larger, the corresponding signal intensity gradually decreases. As the bubbles grow larger, the attenuation of acoustic signals gradually increases. The average acoustic signal strength in still water is $11.64 \mathrm{~dB}$. The average acoustic signal strength in small bubbles is $-19.26 \mathrm{~dB}$. The average acoustic signal strength in middle bubbles is $-26.14 \mathrm{~dB}$. The average acoustic signal strength in big bubbles is $-32.03 \mathrm{~dB}$.

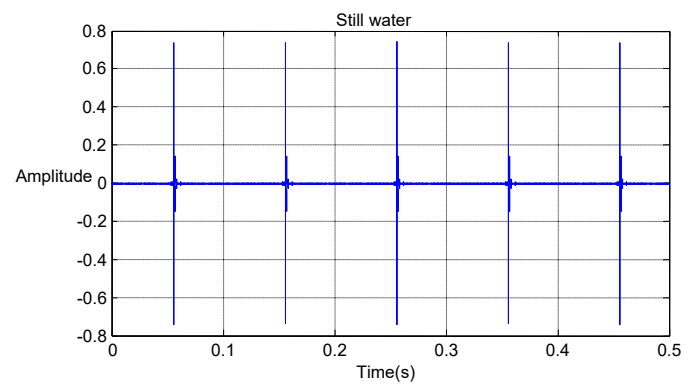

Fig.2. Acoustic signal in still water

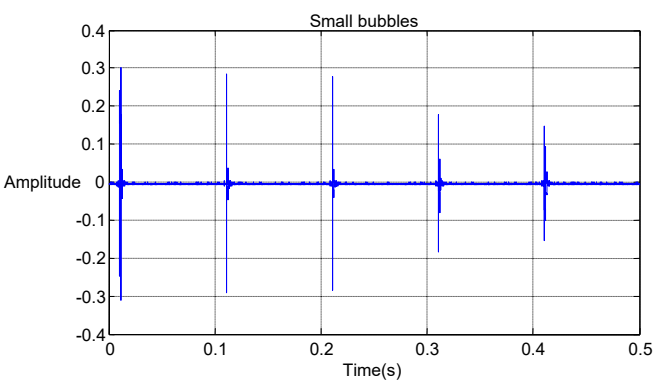

Fig.3. Acoustic in small bubbles

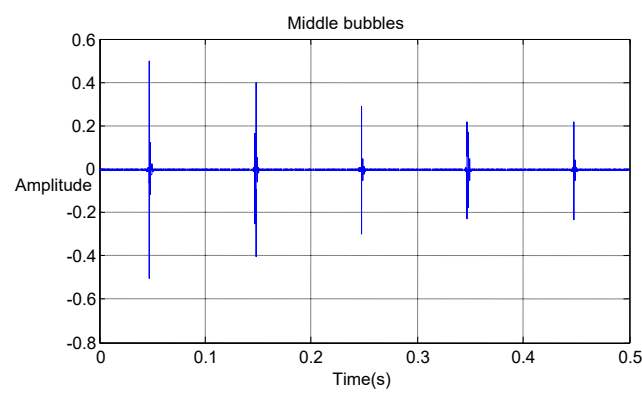

Fig.4. Acoustic in middle bubbles

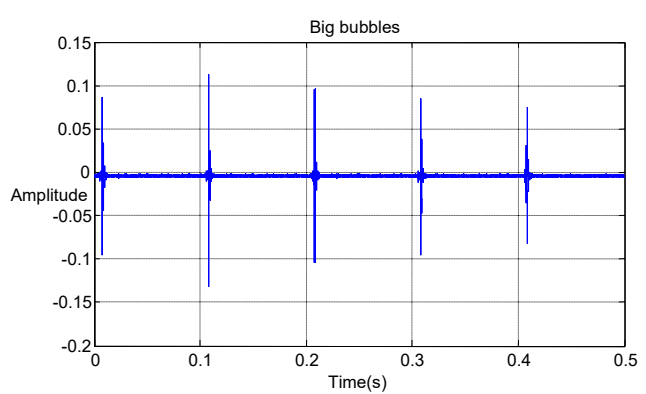

Fig.5. Acoustic in big bubbles

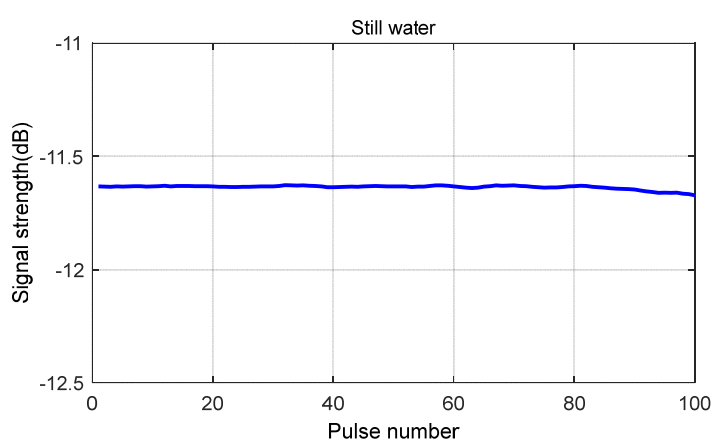

Fig.6. Acoustic signal strength in still water

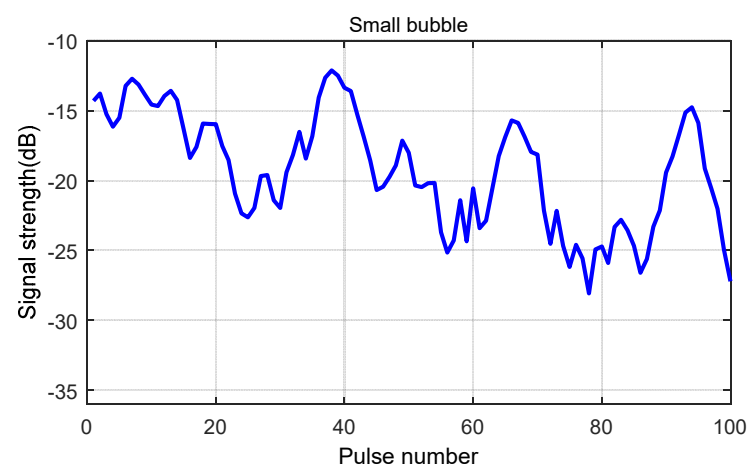

Fig.7. Acoustic signal strength in small bubbles

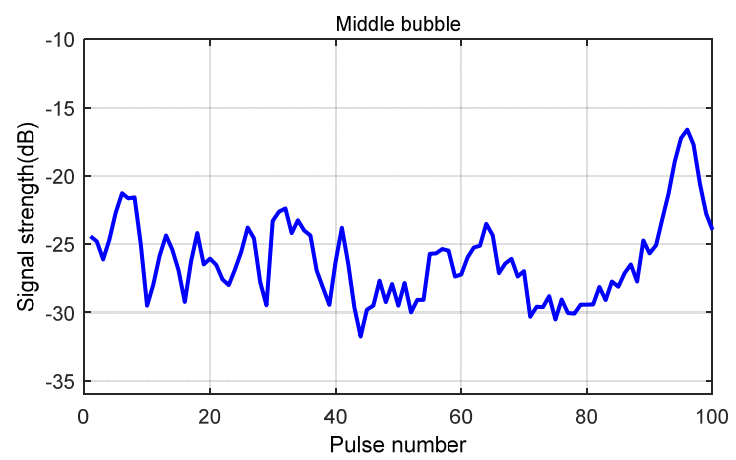

Fig.8. Acoustic signal strength in middle bubbles 


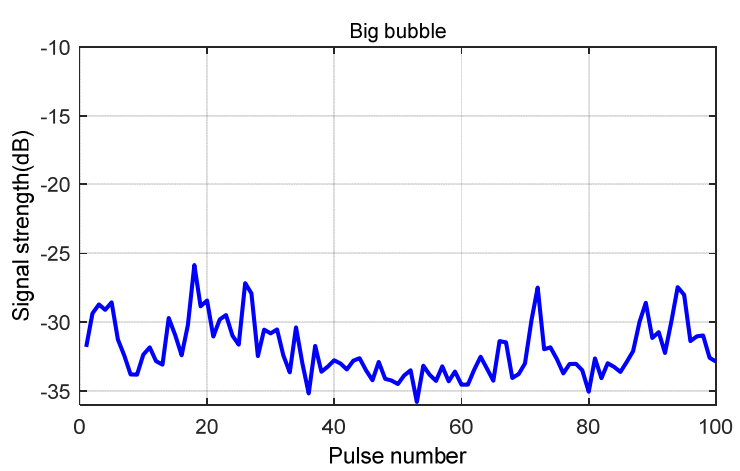

Fig.9. Acoustic signal strength in big bubbles

The sound signals received by the hydrophone under the conditions of still water, small bubble, medium bubble and large bubble were processed. The sound attenuation coefficient is calculated according to the distance of the acoustic signal propagation. The calculated results for different frequencies were shown in table.1. From the analysis of the results, it can be obtained that with a certain frequency, the larger the bubble size, the greater the sound attenuation coefficient. When the bubble size is constant, the sound attenuation coefficient in small bubbles varies greatly below $50 \mathrm{kHz}$. While that above $50 \mathrm{kHz}$, it varies relatively gently with little fluctuation. For medium and large bubbles, the sound attenuation coefficient fluctuates more than that in small bubbles. Whether it is small bubble, medium bubble or large bubble, the sound attenuation coefficient of LFM signal is smaller than that of single frequency $\mathrm{CW}$ signal. And the fluctuation of single frequency signal is large. The fluctuation of LFM signal is suppressed to some extent due to the certain bandwidth of LFM signal.

Table1. Relation of sound attenuation coefficient with bubble and frequency

\begin{tabular}{|c|c|c|c|}
\hline $\begin{array}{c}\text { frequency } \\
(\mathrm{kHz})\end{array}$ & $\begin{array}{c}\text { Small } \\
\text { bubble } \\
(\mathrm{dB} / \mathrm{m})\end{array}$ & $\begin{array}{c}\text { Medium } \\
\text { bubble } \\
(\mathrm{dB} / \mathrm{m})\end{array}$ & $\begin{array}{c}\text { Big } \\
\text { bubble } \\
(\mathrm{dB} / \mathrm{m})\end{array}$ \\
\hline 20 & 3.9027 & 3.0892 & 5.6486 \\
\hline 30 & 4.3730 & 6.0081 & 6.3162 \\
\hline 40 & 4.8892 & 4.8865 & 5.5162 \\
\hline $20-40$ & 3.7541 & 4.5595 & 5.8297 \\
\hline 50 & 2.8027 & 2.9297 & 4.9892 \\
\hline 60 & 2.7486 & 2.9568 & 4.8919 \\
\hline 70 & 3.4378 & 3.1703 & 5.5838 \\
\hline 80 & 3.2973 & 2.8243 & 6.1703 \\
\hline $40-80$ & 2.7378 & 3.1811 & 5.0081 \\
\hline 100 & 3.0568 & 4.3784 & 5.7378 \\
\hline 120 & 3.5811 & 4.1784 & 5.4946 \\
\hline 150 & 3.5622 & 3.3297 & 6.0676 \\
\hline $100-200$ & 2.0595 & 3.9189 & 5.5108 \\
\hline
\end{tabular}

Formula (1) is the integral relation between the sound attenuation coefficient and the bubble. Given the bubble distribution, the sound attenuation coefficient of different incident frequencies can be obtained through formula (1).

$$
\alpha=4 \pi \int_{0}^{\infty} \frac{\mathrm{R}^{2} n(R)(\delta / k R)}{\left(\frac{f_{r}^{2}}{f^{2}}-1\right)^{2}+\delta^{2}} d R
$$

$\alpha$ is the acoustic attenuation coefficient. $\mathrm{R}$ is the bubble radius. $n(R)$ is the number of bubbles with radius $\mathrm{R}$ in water per unit volume. $f_{r}$ is the resonance frequency. $f$ is the incident frequency of sound wave. $k$ is the wave number and $\delta$ is the attenuation constant.

The sound attenuation coefficient is calculated under the condition that the bubble radius is $300 \mu \mathrm{m}$ and the number density is $3 \times 10^{6}$. The calculated results are compared with the measured sound attenuation coefficient, as shown in figure.2. The solid green line in the figure.10 is the sound attenuation coefficient calculated theoretically. The blue + line in the figure. 10 is the sound attenuation coefficient measured in small bubble. The red * line in the figure. 10 is the sound attenuation coefficient measured in medium bubble. The black o line in the figure. 10 is the sound attenuation coefficient measured in big bubble.

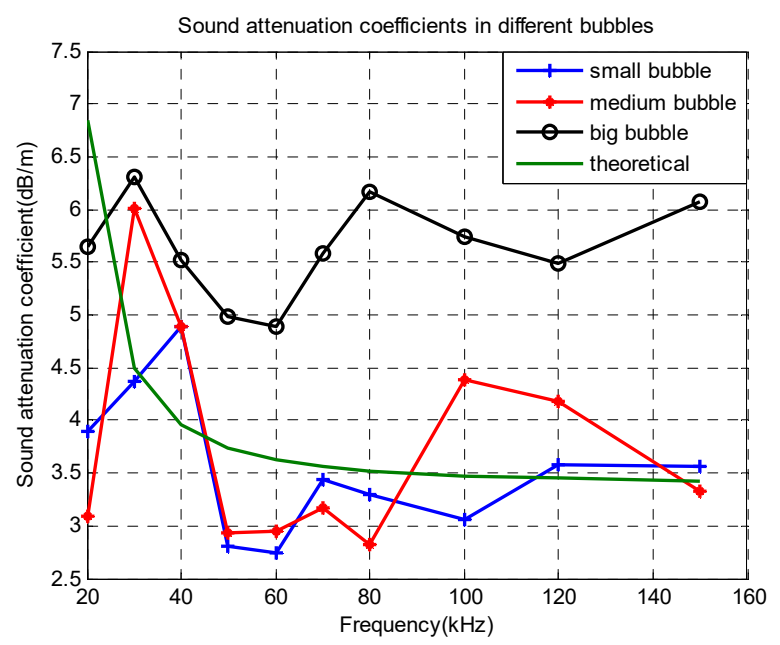

Fig.10. Comparison of sound attenuation coefficients in different bubbles

According to the comparison in the figure.10, the following results can be obtained. The sound attenuation coefficient is large at low frequency. As the frequency increases, the sound attenuation coefficient in the bubble decreases gradually. And the decreasing trend gradually slows down. When the bubble size is certain, the acoustic decay coefficient in the bubble decreases with the increase of frequency. The smaller the bubble is, the smaller the fluctuation is. When the frequency is greater than $50 \mathrm{kHz}$, the average sound attenuation coefficient in the small bubbles measured in the pool test is about $3 \mathrm{~dB} / \mathrm{m}$. It is basically consistent with the theoretical 
calculation results. The formula assumes that the bubble radius and the number density are constant. It can be seen that the variation trend of sound attenuation coefficient measured by the pool experiment is basically the same as that obtained by the theoretical calculation, which verifies the correctness of the above formula of sound attenuation coefficient in bubble wake.

\section{Conclusions}

In this paper, the sound attenuation coefficient under the conditions of still water and different bubble size is measured by pool experiment. The transducer emits acoustic signals of different frequencies in the experiment. The following conclusions are obtained through experiments. The sound attenuation coefficient is large at low frequency. The sound attenuation coefficient in the bubble decreases gradually as the frequency increases. And the decreasing trend gradually slows down. The acoustic decay coefficient in the bubble decreases with the increase of frequency when the bubble size is certain. The smaller the bubble is, the smaller the fluctuation is. Average sound attenuation coefficient in the small bubbles measured in the pool test is about $3 \mathrm{~dB} / \mathrm{m}$ when the frequency is greater than $50 \mathrm{kHz}$. It is basically consistent with the theoretical calculation results.

\section{Acknowledgments}

This research work was supported by the fund program under Grant No. 6142407040104.

\section{References}

1. R.L. Field, E. Jarosz, J.N. Mourn, Acoustic propagation in turbulent layers, Conference Title: Oceans2007, pp280-286(2007).

2. X.B. Tian, J.N. Gu, Z.H. Zhang, C. Wang, Effect of ship speed on Bubble wake geometry characters, Torpedo Technology, 18:268-271(2010).

3. P.G.Bergmann et al, Fundamentals of hydroacoustic physics, Science Press, 592-600(1959).

4. J. Gao, J.Y. Zhang and L.Yang, The present situation of research on ship wake characteristic, Ship Science and Technology, 30:27-32(2008).

5. Lalentin, L, Anatoliy, S and John H.P., Sound velocity and attenuation in bubbly gels measured by transmission experiments, J Acoust. Soc. Am 123:1931-1940(2008).

6. Teng Y.H., Song Y, Li S.W., The research on bubble density spectrum estimation of wake, 32:182-185(2013). 